\title{
Effect of football boot upper padding on shooting accuracy and velocity performance
}

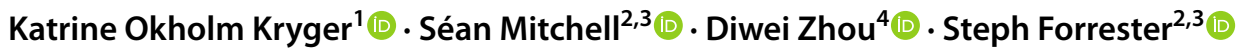

(c) The Author(s) 2020

\begin{abstract}
Football boots are marketed with a specific performance feature focus, for example, power boots are marketed for optimal shooting performance. However, little evidence exists on the impact of boot design on shooting performance. This study assessed the effect of upper padding on shooting velocity and accuracy using a test-retest reliable test setup. Nine university level football players performed a protocol of shooting to: (1) maximise velocity; and (2) maximise accuracy in football boots with and without upper padding (Poron Memory foam). The protocol was completed twice; the non-padded boot results were used for test-retest validation, while the non-padded versus padding results were used to investigate the effect of padding. Velocity was assessed through actual ball velocity, percentage of maximum velocity and perceived velocity. Accuracy was assessed through radial offset, vertical offset, horizontal offset, success (goal/no goal), zonal offset and perceived accuracy. No significant differences between boots were observed in the velocity measures for either velocity or accuracy focused shots. Significant differences between boots were observed in vertical offset for both accuracy (without padding mean \pm standard deviation $-0.02 \pm 1.05 \mathrm{~m}$, with padding $0.28 \pm 0.87 \mathrm{~m}, P=0.029$ ) and velocity (without padding $0.04 \pm 1.33 \mathrm{~m}$, with padding $0.38 \pm 0.86 \mathrm{~m}, P=0.042$ ) focused shots resulting in more missed shots above the goal for the padded boot (without padding 41-43\% missed, with padding 56-72\% missed). These findings suggest the addition of upper padding has a negative impact on shooting accuracy while not impacting shooting velocity.
\end{abstract}

Keywords Soccer $\cdot$ Precision $\cdot$ Kicking $\cdot$ Footwear $\cdot$ Shoe $\cdot$ Speed

\section{Introduction}

Technological innovations are frequently introduced by sporting goods companies in an increasingly competitive and continually changing global football footwear market as a means to distinguish themselves [1]. Football boots tend to be marketed on one key performance characteristic (e.g., shooting power). Despite the advertised performance benefits, little research has been published on how design parameters impact a player's performance along with reliable means to assess this.

This article is a part of Topical Collection in Sports Engineering on Football Research, edited by Dr. Marcus Dunn, Mr. Johsan Billingham, Prof. Paul Fleming, Prof. John Eric Goff and Prof. Sam Robertson.

Katrine Okholm Kryger

K.Kryger@qmul.ac.uk

Extended author information available on the last page of the article
Shooting performance in football is determined by two key factors: how fast the ball moves and how accurately it reaches its target. For ball velocity, studies have assessed either the general impact of using different marketed boot designs [2-5] or the impact of specific boot parameters [6-10]. Studies utilising different marketed boot designs have reported conflicting results; significant differences [2] and no significant differences [4] in ball velocity have been reported, while others did not include any statistical comparison of ball velocity $[3,5]$. With little control over the specific design feature differences between the marketed boot designs used, these conflicting results are perhaps to be expected. In more controlled studies, boot mass has been found to have no effect on ball velocity [6-8]. An increased toe box height has been found to reduce ball velocity by up to $2 \%$ [8], but it is unclear whether toe box height was the only design parameter that differed between the boots used. Upper friction, defined as 'low friction', 'regular friction', 'high friction' and 'very high friction' (with no upper material frictional properties included), demonstrated 
tendencies towards higher ball velocities using the 'regular friction' upper $(P \leq 0.07)$ [9], but without quantifying the upper material frictional properties these results have limited practical significance. In a comparison of soft ground, firm ground and two trimmed length (50 and $0 \%$ of original stud length) studs on artificial turf [10] significantly higher ball velocities were obtained for the firm ground and 50\% length studs, suggesting that stud length and, thereby, traction can affect a player's ability to generate ball velocity.

Fewer studies have assessed shooting accuracy. Hennig et al. [11] compared radial offset from target for five boots and a barefoot condition with significant differences of up to $13 \%$ observed. Kuo and Shang [12] investigated the effect of lace location on radial offset, with laces located either 'inside' or 'outside' found to produce significantly more accurate shot outcomes compared to designs defined as 'lace on the side' and 'laces cowered'. It is unclear whether lace location was the only difference between the boots assessed.

None of the above-mentioned studies applied a test-retest validated protocol. Moreover, all of these studies attempted to assess either ball velocity or radial offset from a target and not both together. The speed-accuracy trade-off theory by Fitts [13] claims that focusing on one of these two parameters will impact the outcome of the other. The speedaccuracy trade-off theory has been demonstrated in football shooting with increased ball velocity leading to decreased ball accuracy and vice versa [14]. Another limitation is how offset was assessed. All studies used a radial offset measure; direction of offset was not considered despite its importance in football potentially differentiating between a goal and no goal. Protocols assessing shooting performance with a focus on the players' skill level have previously been validated [15, 16]. Ali et al. [15] applied a point system for marked scoring zones to assess accuracy, which is difficult to contextualise and, therefore, limits the practical application. The study also lacked a measure of ball velocity, and therefore, the speed-accuracy trade-off may have impacted performance. Russell et al. [16] assessed radial offset using two-dimensional (2-D) video analysis, assessing the goal from a single frontal facing camera. It is unclear how the frame corresponding to the ball crossing the goal line was identified for assessment of radial offset. Furthermore, the assessment was based on $50-60 \mathrm{~Hz}$ video resulting in large frame-to-frame ball displacements (e.g., at $22 \mathrm{~m} \mathrm{~s}^{-1}$ the ball moves $0.37-0.44 \mathrm{~m}$ between frames).

Football boots marketed as shooting performance focused have, since their introduction in 2013, been designed with the distinguishing feature of additional upper padding (e.g., Puma EvoPower, released in January 2014). The effect of added upper padding on shooting performance has yet to be investigated within the scientific literature. Thus, the primary aim of this study was to assess the effect of football boot upper padding on shooting performance (i.e., ball velocity and accuracy). The secondary aim was to assess the test-retest reliability of the protocol used. The setup was structured to be easy to apply and demand no more than two researchers to run yet be ecologically valid and produce transferable results. A better understanding of design requirements for optimal shooting would benefit both the industry and consumer.

\section{Methods}

\subsection{Participants}

Nine skilled male football players (age mean \pm standard deviation $22.8 \pm 2.1$ years, stature $1.77 \pm 0.03 \mathrm{~m}$, mass $71.1 \pm 4.5 \mathrm{~kg}$ ) were recruited from the University $1 \mathrm{st}$ football and futsal teams competing in the top division of the British Universities league. All futsal players had a history as a football player prior to University and all players recruited had $9 \pm 4$ year experience of club level football. The number of participants was selected based on similar within-participant assessment style football shooting studies [16, 17]. None of the participants had suffered from match-preventive lower limb injuries in 6 months prior to testing. All participants were UK size 8 and right foot dominant, which was determined by asking participants which side they preferred for kicking. During the test, participants wore the same brand of new football socks (Umbro Pro Tech Ankle socks) to prevent the socks from altering the participants' sensation of the boot and ball. The study received ethical clearance from the institutional ethics committee (Ethics Number: G16-P5) and each participant provided written informed consent in accordance with the requirements of the Helsinki Declaration for research using human participants.

\subsection{Football boots}

UK size 8 prototype football boots were developed by Umbro for the test (Fig. 1). Fit was ensured from verbal feedback and palpation prior to testing. The boots had a similar smooth white synthetic upper, central lacing and a black firm ground outsole similar to the Umbro UX2. Mass of the unpadded boot was $0.211 \mathrm{~kg}$ and of the padded boot $0.216 \mathrm{~kg}$. The boots only differed in upper padding thickness; one boot had no padding and the other had $8 \mathrm{~mm}$ of Poron memory foam padding, which was added during the manufacturing process (Fig. 1). Boot upper hardness was assessed on a size UK8 foot last using a digital Shore Durometer A Scale (range 0-100 HA, resolution 0.5 HA). Shore A at superior toe region of the unpadded boot was $66.2 \pm 0.8 \mathrm{HA}$, while the padded boot was $60.0 \pm 1.6$ $\mathrm{HA}$ and the central instep region of the unpadded boot was $58.5 \pm 0.4 \mathrm{HA}$, while the padded boot was $46.3 \pm 0.6 \mathrm{HA}$. 

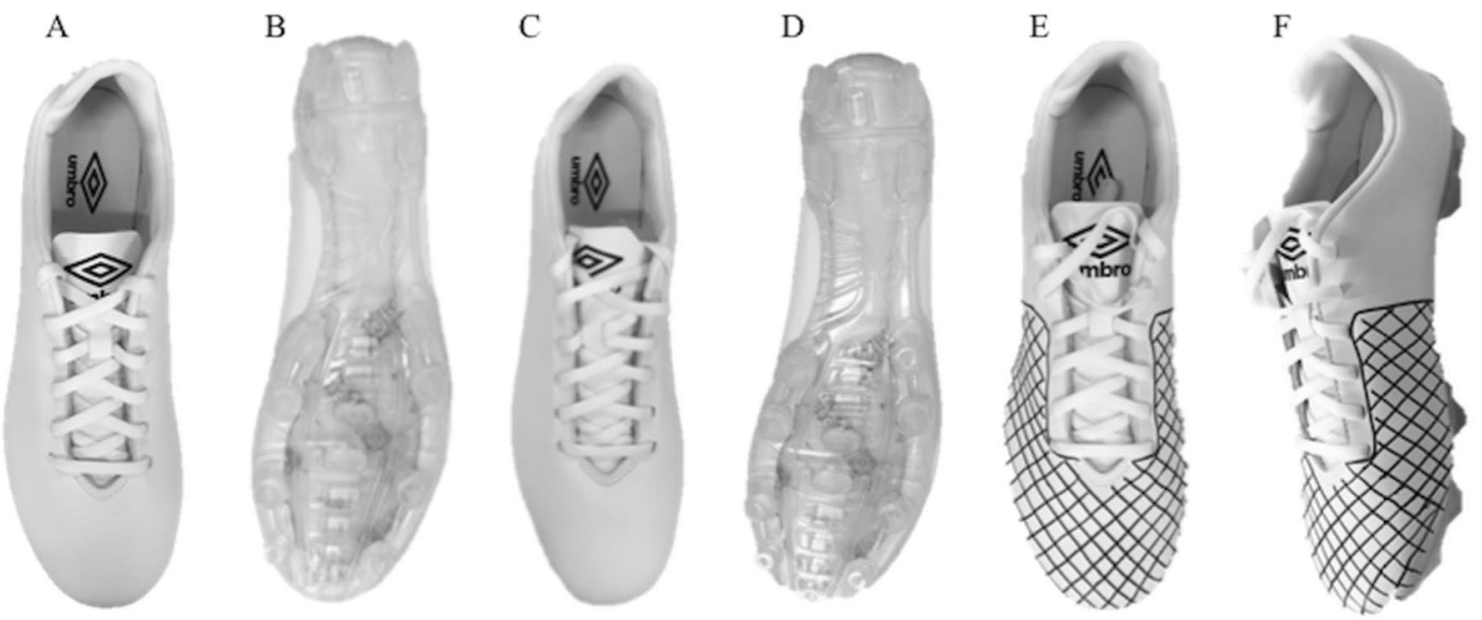

Fig. 1 Plantar and dorsal views of the (a, b): non-padded boot; and $\mathbf{c}-\mathbf{f}$ Poron Memory foam padded boot, where $\mathbf{e}, \mathbf{f}$ is the dorsal and medial view with the upper padding zone highlighted

Poron memory foam padding has previously been used for padding in football boot designs [18]. The experimental design limited the study to two thickness conditions without fatigue becoming an issue, while the study aim indicated that these should represent the extremes of what might be practical to include in football boots. The boots were masked such that the participants were not informed of any differences between boots, i.e., a blind study.

\subsection{Experimental design}

The study followed the Okholm Kryger et al. [19] study design with test-retest and boot comparison merged together. Players participated in pairs to allow peer competition as a motivative factor. The pairs took part in two sessions of $2 \mathrm{~h}$ duration separated by 7-14 days. Each session comprised a standardised warm up and familiarisation of each drill performed in the player's own football boots. Thereafter, the main test session comprised 10 shots focused on shooting accuracy in each boot (randomised order) followed by 10 shots focused on maximum ball velocity in each boot (randomised order)—totalling 40 shots per participants. The two players alternated every 10 shots to minimise fatigue.

Tests were performed on the same goal on an outdoor third generation artificial pitch (LigaTurf RS + CoolPlus 260, Polytan, Burgheim, Germany). In brief, the pitch had a $25 \mathrm{~mm}$ in situ rubber shock pad, the carpet fibres were $60 \mathrm{~mm}$ monofilament polyethylene and the infill comprised $15 \mathrm{~kg} \mathrm{~m}^{-2}$ sand and $15 \mathrm{~kg} \mathrm{~m}^{-2}$ rubber crumb giving a total infill height of $41 \mathrm{~mm}$. Pitch testing conducted immediately after this study using the FIFA Quality Concept methodologies [20], gave a force reduction of $69.6 \pm 1.5 \%$, vertical deformation of $11.4 \pm 0.5 \mathrm{~mm}$ and rotational resistance of
$31.9 \pm 1.3 \mathrm{Nm}$. Tests were only performed under dry and not windy conditions.

An Adidas miCoach football (Adidas, Herzogenaurach, Germany) (diameter $=0.220 \mathrm{~m}$, mass $=0.43 \mathrm{~kg}$, pressure $=0.9$ bar) was used for the tests. Pressure was tested before and after each session with no measurable change during the session. The Adidas miCoach football was placed with the manufacturer specified orientation for each shooting instance (valve facing kicker; middle arrow facing towards centre of target). Using the same target point on the ball for each shot limited the risk of the ball impacting the results $[21,22]$.

The ball was placed for a free kick scenario $16 \mathrm{~m}$ from the goal and directly behind the penalty spot (Fig. 2). Players used a repeated but self-selected run up. The test shots were used to determine their preferred run up pattern for accuracy and another set of test shots were used to determine their preferred run up pattern for maximum ball velocity shots. The start position was marked with a cone and participants were instructed to repeat the same run up for every shot. The start positions were recorded and used again for the repeated session to prevent the run up impacting shooting performance [23, 24]. The top right corner of the goal was used as the target point for both accuracy and velocity focused shots and was approximated as $0.11 \mathrm{~m}$ lower than the bar and $0.11 \mathrm{~m}$ inside the post, based on the ball radius being $0.11 \mathrm{~m}$ ). This target ball location in the top corner has been identified as the best one to beat a goalkeeper when shooting [15].

To enhance ecological validity yet respecting that changing technique can impact shooting performance [25], players were instructed to self-select but apply the same kicking technique for every shot. Boot-ball impact was recorded using a single high-speed video (CASIO EX-FH1000, Casio 


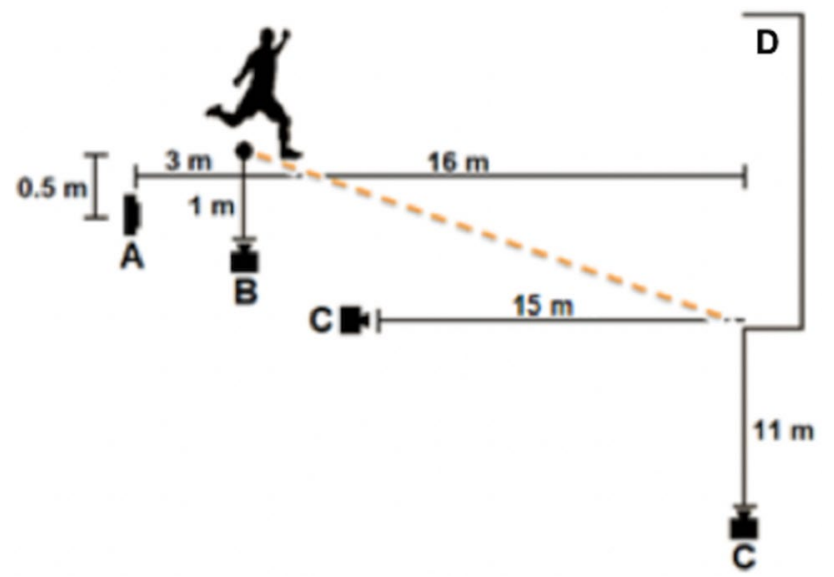

Fig. 2 Aerial view of test setup. Note: Ball placed $16 \mathrm{~m}$ in front of centre of goal; Shots target top right corner of the goal; $A$ TrackMan Football; $B$ CASIO EX-FH1000 camera; $C$ two GoPro HERO4 Black cameras; $D$ goal

Computer Co., Tokyo, Japan; 420 Hz, $230 \times 170$ pixels; barrel distortion $\leq 0.1 \%$ ). Within-participant consistency in technique was visually assessed and any shot varying in technique was excluded.

Ball velocity was assessed with TrackMan Football (TrackMan Golf, Vedbaek, Denmark) placed $3 \mathrm{~m}$ behind the ball with a $0.5 \mathrm{~m}$ offset at the opposite site to the run up (e.g., right for right-footed players). The system has previously been validated for ball velocity assessment in football (Bland-Altman mean difference of $-0.46 \mathrm{~m} \mathrm{~s}^{-1}$ and limits of agreement of -2.34 to $1.42 \mathrm{~m} \mathrm{~s}^{-1}$ when compared to a Vicon MX motion analysis system capturing at $400 \mathrm{~Hz}$ ) [26]. Offset from target was recorded using two GoPro HERO4 Black cameras $(240 \mathrm{~Hz}, 1280 \times 720$ pixels; GoPro Inc., San Mateo, CA). One camera, rotated $90^{\circ}$ to allow portrait view, was placed $9 \mathrm{~m}$ from the goal post, $1 \mathrm{~m}$ above the ground, and along the back line to identify the moment when the ball passed the goal line (Fig. 2). The second camera was placed $15 \mathrm{~m}$ directly in front of the target, and at the height of the target to capture ball offset from the target when the goal passed the ball line (Fig. 2). A spirit level was used to ensure the camera was level and a laser was used to ensure it was facing the target point. Pilot studies demonstrated a pixel size of $0.008 \mathrm{~m} \mathrm{pixel}^{-1}$ and a maximal barrel distortion of $-2.1 \%$. The two cameras were controlled wirelessly by a GoPro Smart Remote (GoPro Inc., San Mateo, CA). A manufactured light sync device, with four light columns changing at different speeds $(1=1 \mathrm{~Hz}, 2=10 \mathrm{~Hz}, 3=100 \mathrm{~Hz}$, $4=1000 \mathrm{~Hz}$ ) was placed in the vision of both cameras to allow synchronisation of the images.

To assess participants' subjective perception of performance, a questionnaire was included. Participants completed a 7-point Likert scale after each shot evaluating the player's perceived performance. For accuracy shots, participants were asked to score the accuracy of their shots ( $1=$ 'extremely accurate' to $7=$ 'extremely inaccurate'), while for maximum velocity shots, participants were asked to score the ball speed achieved $(1=$ 'extremely fast' to $7=$ 'extremely slow').

\subsection{Analysis of measures}

Maximum ball velocity measures were presented as velocity in $\mathrm{m} \mathrm{s}^{-1}$ as well as percentage of the player's fastest shot, as maximum ball velocity is relative to the individual.

For accuracy assessment, the frame where the centre-line of the ball passed over the goal line was defined from the camera placed on the goal line. The equivalent frame from the camera placed in front of the goal was used for analysis of shot accuracy. This frame was manually analysed in Image-Pro Analyzer (Version 7.0, Media Cybernetics, Inc., Rockville, MD). Ball location was defined as the centre point of the ball $(x, y)$ obtained from the Best Fit Circle function using at least five digitised points on the ball circumference. The target location was defined by recording the ball statically placed at the defined target point (extreme top right corner of the goal) at the start and end of each test session. The two recordings were performed to ensure no camera movement during the session. The ball location, target location and ball radius were extracted from Image-Pro Analyser as pixels and converted to metres through the known ball diameter $(0.220 \mathrm{~m})$. Accuracy was measured as radial offset, vertical offset ( $y$-axis offset; Fig. 3), horizontal offset ( $x$-axis offset; Fig. 3), zonal offset spread (Fig. 3) and success (goal/ no goal).

\subsection{Statistical analysis}

Statistical analysis was carried out using SPSS software (Version 23.0; SPSS Inc., Chicago, IL). All data is presented at mean \pm standard deviation unless stated otherwise. Statistical significance was set at $P \leq 0.05$. Data for the boot comparison was selected from session one and hence comparing within session measures. The independent variable of upper padding and dependent variables ball velocity measures (actual and percentage of maximum ball velocity achieved on an individual player basis), accuracy measures (radial offset, vertical offset, horizontal offset and variance of these measures using participant standard deviations) and subjective performance measures were assessed using oneway MANOVAs for accuracy and velocity focused shots. Success was assessed using Fischer's exact test.

To assess the test-retest reliability of the protocol, relative and absolute reliability of the performance variables were examined. The magnitude of relative reliability was determined by the two-way random effect intraclass correlation 


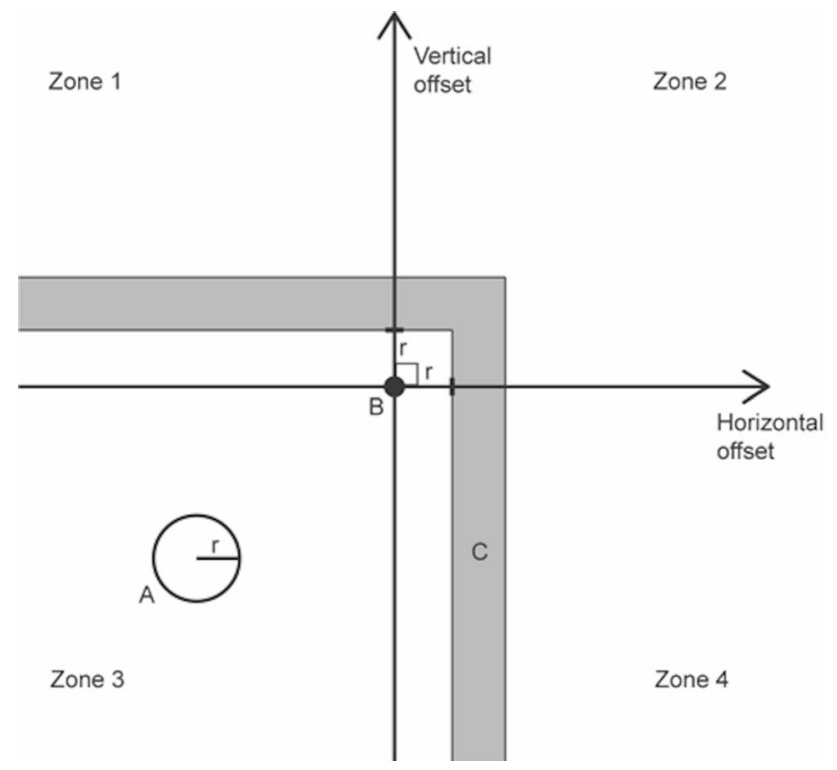

Fig. 3 Zones used to organise offset of shots. Four zones were created through placing a vertical line and a horizontal line intersecting at the target point. $A$ football with radius ' $r$ '; $B$ target point with $r$ distance to goal post and crossbar; $C$ goal post and bar; radial offset $=$ distance $A_{\text {centre }}-B$. Zone 3 indicates a goal scored

coefficient $\left(\mathrm{ICC}_{2,1}\right.$; absolute agreement definition) using the mean participant scores for each of the two non-padded boot sessions [27]. Values were interpreted based on the clinical significance levels suggested by Cicchetti [28]. Data was log-transformed due to heteroscedasticity as suggested by Vaz et al. [29] and Weir [27]. Absolute reliability was assessed using standard error of measurement (SEM) and the smallest real difference (SRD) derived from the intra-class correlation coefficients following the methods explained by Weir [27].

\section{Results}

There was a statistically significant difference between upper padding conditions for ball velocity and accuracy measures in both accuracy focused kicks $(F(8,9)=13.61, P<0.001$; Wilk's $\Lambda=0.076$, partial $\left.\eta^{2}=0.924\right)$ and velocity focused kicks $(F(8,9)=3.73, P=0.033$; Wilk's $\Lambda=0.232$, partial $\left.\eta^{2}=0.768\right)$.

\subsection{Effect of upper padding on shooting ball velocity}

Ball velocity assessed as percentage of maximum and actual velocity showed no significant difference between upper padding conditions for both accuracy focused shots (Percentage: non-padded $72.6 \pm 7.7 \%$, padded $72.9 \pm 7.4 \%, P=0.933$;
Actual velocity: non-padded $22.7 \pm 2.6 \mathrm{~m} \mathrm{~s}^{-1}$, padded $\left.22.6 \pm 2.8 \mathrm{~m} \mathrm{~s}^{-1}, P=0.898\right)$ and velocity focused shots (Percentage: non-padded 92.2 $\pm 2.8 \%$, padded $93.0 \pm 2.6 \%$, $P=0.673$; Actual velocity: non-padded $28.9 \pm 1.9 \mathrm{~m} \mathrm{~s}^{-1}$, padded $29.0 \pm 1.8 \mathrm{~m} \mathrm{~s}^{-1}, P=0.707$; Table 1) despite demonstrating moderate relative reliability (Percentage: $\mathrm{ICC}_{2,1}=0.640 ;$ Actual velocity: $\mathrm{ICC}_{2,1}=0.537$; Table 1) and absolute reliabilities (SRD) of $\pm 10.1 \%$ for percentage defined ball velocity and $3.2 \mathrm{~m} \mathrm{~s}^{-1}$ for actual ball velocities.

\subsection{Effect of upper padding on shooting accuracy}

Likewise, no significant difference was found for radial offset outcomes between the two upper padding conditions for accuracy focused shots (non-padded $1.76 \pm 0.90 \mathrm{~m}$, padded $\left.1.86 \pm 0.48 \mathrm{~m}, P=0.439 ; \mathrm{ICC}_{2,1}=0.824 ; \mathrm{SRD}=0.58 \mathrm{~m}\right)$ or velocity focused shots (non-padded $2.39 \pm 1.17 \mathrm{~m}$, padded $2.96 \pm 0.86 \mathrm{~m}, P=0.182 ; \quad \mathrm{ICC}_{2,1}=0.717$; $\mathrm{SRD}=1.06 \mathrm{~m}$; Table 1). Similarly, no difference was seen for the horizontal offset for accuracy focused shots (nonpadded $-1.13 \pm 1.24 \mathrm{~m}$, padded $-1.02 \pm 1.63 \mathrm{~m}, P=0.767$; $\left.\mathrm{ICC}_{2,1}=0.900 ; \mathrm{SRD}=0.55 \mathrm{~m}\right)$ or velocity focused shots (non-padded $-1.60 \pm 1.68 \mathrm{~m}$, padded $-1.96 \pm 1.52 \mathrm{~m}$, $P=0.182 ; \mathrm{ICC}_{2,1}=0.612 ; \mathrm{SRD}=1.54 \mathrm{~m}$; Table 1). Vertical offset was, however, significantly greater (ball above the bar) for shots performed in the padded boot for both accuracy focused shots (non-padded $-0.02 \pm 1.05 \mathrm{~m}$, pad$\left.\operatorname{ded} 0.28 \pm 0.87 \mathrm{~m}, P=0.038 ; \mathrm{ICC}_{2,1}=0.653 ; \mathrm{SRD}=0.70 \mathrm{~m}\right)$ and velocity focused kicks (non-padded $0.04 \pm 1.33 \mathrm{~m}$, pad$\operatorname{ded} 0.38 \pm 0.86 \mathrm{~m}, P=0.046 ; \mathrm{ICC}_{2,1}=0.714 ; \mathrm{SRD}=0.73 \mathrm{~m}$; Table 1).

Variance of radial offset was significantly greater in the padded boot during velocity focused shots (nonpadded $0.86 \pm 0.38 \mathrm{~m}$, padded $1.31 \pm 0.41 \mathrm{~m}, P=0.026$; $\mathrm{ICC}_{2,1}=0.468 ; \mathrm{SRD}=1.13 \mathrm{~m}$; Table 1 ), while no difference was seen for accuracy focused shots (non-padded $0.84 \pm 0.27 \mathrm{~m}$, padded $0.73 \pm 0.16 \mathrm{~m}, P=0.290$; $\mathrm{ICC}_{2,1}=0.496 ; \mathrm{SRD}=0.42 \mathrm{~m}$; Table 1). No difference was seen for variance of horizontal offset for accuracy focused kicks (non-padded $1.07 \pm 0.41 \mathrm{~m}$, padded $1.06 \pm 0.36 \mathrm{~m}$, $P=0.945 ; \mathrm{ICC}_{2,1}=0.807 ; \mathrm{SRD}=0.0 .61 \mathrm{~m}$; Table 1$)$ nor velocity focused kicks (non-padded $1.08 \pm 0.47 \mathrm{~m}$, padded $1.57 \pm 0.81 \mathrm{~m}, \mathrm{P}=0.182 ; \mathrm{ICC}_{2,1}=0.551 ; \mathrm{SRD}=1.20 \mathrm{~m}$; Table 1). Variance of vertical offset for accuracy focused kicks was significantly greater in the non-padded boot for accuracy focused shots (non-padded $1.12 \pm 0.37 \mathrm{~m}$, padded $0.85 \pm 0.24 \mathrm{~m}, P=0.048 ; \mathrm{ICC}_{2,1}=0.684 ; \mathrm{SRD}=0.66 \mathrm{~m}$; Table 1), while no difference was seen for velocity focused shots (non-padded $1.19 \pm 0.42 \mathrm{~m}$, padded $1.18 \pm 0.41 \mathrm{~m}$, $P=0.238 ; \mathrm{ICC}_{2,1}=0.617 ; \mathrm{SRD}=0.59 \mathrm{~m}$; Table 1$)$. 
Table 1 Ball velocity and accuracy results for the $0 \mathrm{~mm}$ and $8 \mathrm{~mm}$ padded boots in session 1 and relative and absolute test-retest reliability scores for repeated $0 \mathrm{~mm}$ padded boot test sessions

\begin{tabular}{|c|c|c|c|c|c|c|c|c|c|}
\hline \multirow{3}{*}{ Skill } & \multirow{3}{*}{ Variable } & \multirow{3}{*}{ Boot } & \multicolumn{2}{|c|}{ Padding comparison } & \multicolumn{5}{|c|}{ Padding comparison } \\
\hline & & & Mean \pm SD & $P$ value & Session 1 & Session 2 & $\mathrm{ICC}_{2,1}$ & SEM & SRD \\
\hline & & & & & Mean \pm SD & Mean \pm SD & & & \\
\hline \multirow[t]{18}{*}{ Accuracy } & \multirow[t]{2}{*}{ Velocity (\%) } & $0 \mathrm{~mm}$ & $72.6 \pm 7.7$ & 0.933 & $72.6 \pm 7.7$ & $72.3 \pm 8.0$ & 0.640 & \pm 3.6 & \pm 10.1 \\
\hline & & $8 \mathrm{~mm}$ & $72.9 \pm 7.4$ & & & & & & \\
\hline & \multirow[t]{2}{*}{ Velocity $\left(\mathrm{m} \mathrm{s}^{-1}\right)$} & $0 \mathrm{~mm}$ & $22.7 \pm 2.6$ & 0.898 & $22.7 \pm 2.6$ & $22.6 \pm 2.1$ & 0.537 & \pm 1.1 & \pm 3.2 \\
\hline & & $8 \mathrm{~mm}$ & $22.6 \pm 2.8$ & & & & & & \\
\hline & \multirow[t]{2}{*}{ Radial offset (m) } & $0 \mathrm{~mm}$ & $1.76 \pm 0.90$ & 0.439 & $1.76 \pm 0.90$ & $1.76 \pm 1.03$ & 0.824 & \pm 0.21 & \pm 0.58 \\
\hline & & $8 \mathrm{~mm}$ & $1.86 \pm 0.48$ & & & & & & \\
\hline & \multirow[t]{2}{*}{ Radial variance (m) } & $0 \mathrm{~mm}$ & $0.84 \pm 0.27$ & 0.290 & $0.84 \pm 0.27$ & $0.90 \pm 0.49$ & 0.496 & \pm 0.15 & \pm 0.42 \\
\hline & & $8 \mathrm{~mm}$ & $0.73 \pm 0.16$ & & & & & & \\
\hline & \multirow[t]{2}{*}{ Horizontal offset (m) } & $0 \mathrm{~mm}$ & $-1.13 \pm 1.24$ & 0.767 & $-1.13 \pm 1.24$ & $-1.22 \pm 1.40$ & 0.900 & \pm 0.20 & \pm 0.55 \\
\hline & & $8 \mathrm{~mm}$ & $-1.02 \pm 1.63$ & & & & & & \\
\hline & \multirow[t]{2}{*}{ Horizontal variance $(\mathrm{m})$} & $0 \mathrm{~mm}$ & $1.07 \pm 0.41$ & 0.945 & $1.07 \pm 0.41$ & $1.08 \pm 0.60$ & 0.807 & \pm 0.24 & \pm 0.61 \\
\hline & & $8 \mathrm{~mm}$ & $1.06 \pm 0.36$ & & & & & & \\
\hline & \multirow[t]{2}{*}{ Vertical offset (m) } & $0 \mathrm{~mm}$ & $-0.02 \pm 1.05$ & $0.038 *$ & $-0.02 \pm 1.05$ & $-0.18 \pm 0.85$ & 0.653 & \pm 0.25 & \pm 0.70 \\
\hline & & $8 \mathrm{~mm}$ & $0.28 \pm 0.87$ & & & & & & \\
\hline & \multirow[t]{2}{*}{ Vertical variance $(\mathrm{m})$} & $0 \mathrm{~mm}$ & $1.12 \pm 0.37$ & $0.048 *$ & $1.12 \pm 0.37$ & $0.98 \pm 0.32$ & 0.684 & \pm 0.24 & \pm 0.66 \\
\hline & & $8 \mathrm{~mm}$ & $0.85 \pm 0.24$ & & & & & & \\
\hline & \multirow[t]{2}{*}{ Perceived accuracy } & $0 \mathrm{~mm}$ & $3.5 \pm 0.9$ & 0.332 & $3.5 \pm 0.9$ & $3.3 \pm 0.6$ & 0.490 & \pm 0.00 & \pm 0.01 \\
\hline & & $8 \mathrm{~mm}$ & $3.1 \pm 0.9$ & & & & & & \\
\hline \multirow[t]{18}{*}{ Velocity } & \multirow[t]{2}{*}{ Velocity (\%) } & $0 \mathrm{~mm}$ & $92.2 \pm 3.8$ & 0.673 & $92.2 \pm 3.8$ & $92.5 \pm 3.5$ & 0.846 & \pm 1.3 & \pm 3.6 \\
\hline & & $8 \mathrm{~mm}$ & $93.0 \pm 2.6$ & & & & & & \\
\hline & \multirow[t]{2}{*}{ Velocity $\left(\mathrm{m} \mathrm{s}^{-1}\right)$} & $0 \mathrm{~mm}$ & $28.9 \pm 1.9$ & 0.707 & $28.9 \pm 1.9$ & $29.0 \pm 1.8$ & 0.944 & \pm 0.4 & \pm 1.2 \\
\hline & & $8 \mathrm{~mm}$ & $29.0 \pm 1.8$ & & & & & & \\
\hline & \multirow[t]{2}{*}{ Radial offset (m) } & $0 \mathrm{~mm}$ & $2.39 \pm 1.17$ & 0.182 & $2.39 \pm 1.17$ & $2.41 \pm 1.14$ & 0.717 & \pm 0.38 & \pm 1.06 \\
\hline & & $8 \mathrm{~mm}$ & $2.96 \pm 0.86$ & & & & & & \\
\hline & \multirow[t]{2}{*}{ Radial variance (m) } & $0 \mathrm{~mm}$ & $0.86 \pm 0.38$ & $0.026^{*}$ & $0.86 \pm 0.38$ & $0.97 \pm 0.41$ & 0.468 & \pm 0.36 & \pm 1.13 \\
\hline & & $8 \mathrm{~mm}$ & $1.31 \pm 0.41$ & & & & & & \\
\hline & \multirow[t]{2}{*}{ Horizontal offset (m) } & $0 \mathrm{~mm}$ & $-1.60 \pm 1.68$ & 0.132 & $-1.60 \pm 1.68$ & $-1.67 \pm 1.61$ & 0.612 & \pm 0.56 & \pm 1.54 \\
\hline & & $8 \mathrm{~mm}$ & $-1.96 \pm 1.52$ & & & & & & \\
\hline & \multirow[t]{2}{*}{ Horizontal variance $(\mathrm{m})$} & $0 \mathrm{~mm}$ & $1.08 \pm 0.47$ & 0.182 & $1.08 \pm 0.47$ & $1.18 \pm 0.37$ & 0.551 & \pm 0.43 & \pm 1.20 \\
\hline & & $8 \mathrm{~mm}$ & $1.57 \pm 0.81$ & & & & & & \\
\hline & \multirow[t]{2}{*}{ Vertical offset (m) } & $0 \mathrm{~mm}$ & $0.04 \pm 1.33$ & $0.046^{*}$ & $-0.04 \pm 1.33$ & $0.06 \pm 1.34$ & 0.714 & \pm 0.26 & \pm 0.73 \\
\hline & & $8 \mathrm{~mm}$ & $0.38 \pm 0.86$ & & & & & & \\
\hline & \multirow[t]{2}{*}{ Vertical variance $(\mathrm{m})$} & $0 \mathrm{~mm}$ & $1.19 \pm 0.42$ & 0.238 & $1.19 \pm 0.42$ & $1.26 \pm 0.36$ & 0.617 & \pm 0.21 & \pm 0.59 \\
\hline & & $8 \mathrm{~mm}$ & $1.18 \pm 0.41$ & & & & & & \\
\hline & \multirow[t]{2}{*}{ Perceived velocity } & $0 \mathrm{~mm}$ & $2.7 \pm 0.5$ & 0.341 & $2.7 \pm 0.5$ & $2.7 \pm 0.7$ & 0.161 & \pm 0.00 & \pm 0.01 \\
\hline & & $8 \mathrm{~mm}$ & $2.6 \pm 0.5$ & & & & & & \\
\hline
\end{tabular}

$I C C_{2,1}$ intraclass correlation coefficient: two-way random effect model (absolute agreement definition), $S D$ Standard deviation, $S E M$ standard error of measurement $=\mathrm{SD} \times \sqrt{1-\mathrm{ICC}}, \mathrm{SRD}$ smallest real difference at $95 \%$ confidence intervals $=\mathrm{SEM} \times 1.96 \times \sqrt{2}$

\subsection{Effect of upper padding on shooting success}

Success, measured by goals scored, was significantly higher for both accuracy and velocity focused shots in the nonpadded football boot (Accuracy Goal: non-padding 58\%, padded $28 \%, P=0.001 ; \mathrm{ICC}_{2,1}=0.925 ; \mathrm{SRD}= \pm 12 \%$; Velocity Goal: non-padded $57 \%$, padded $44 \%, P=0.001$;
$\mathrm{ICC}_{2,1}=0.0 .527 ; \mathrm{SRD}= \pm 20 \%$; Table 2). Of the attempts, $30 \%$ were placed in zone 1 (misses above goal; Fig. 3 ) using the non-padded boot for both accuracy and velocity shots (Fig. 4). While 56\% and $43 \%$ were placed in zone 1 in the padded boot condition, i.e., 26 and $13 \%$ points more than the non-padded boot, the difference being statistically significant 
for accuracy focused shots $(P=0.006$; Table 3$)$ but not for velocity focused shots $(P=0.847$; Table 3$)$.

\subsection{Effect of upper padding on player perception of shooting accuracy and ball velocity}

$\mathrm{ICC}_{2,1}$ was shown to be fair for accuracy perception and poor for velocity perception due to the small range of velocity scores (Table 1). The SRDs did, however, demonstrate an absolute reliability band of SRD $=0.1$ for both perception measures. Despite the small SRD, players' perception of accuracy and ball velocity performance did not significantly differ between the masked upper padding conditions (Accuracy: non-padded 3.5 \pm 0.9 , padded $3.1 \pm 0.9$, $P=0.742 ; \mathrm{ICC}_{2,1}=0.490 ; \mathrm{SRD}= \pm 0.01$; Velocity: non-padded $2.7 \pm 0.5$, padded $2.6 \pm 0.5, P=0.412 ; \mathrm{ICC}_{2,1}=0.161$; $\mathrm{SRD}= \pm 0.01$; Table 1). Interestingly, no players detected the design difference between the two boots. Some verbally reported no difference between boots, while some reported a slightly tighter or stiffer fit in the padded condition.

\section{Discussion}

Ball velocity, assessed as both actual velocity and as a percentage of the maximum velocity achieved by the player, demonstrated no significant differences between upper padding conditions. In agreement, players did not perceive any differences in ball velocity. This outcome does not support the addition of upper padding for performance enhancement, although no negative impact on ball velocity was detected either. Although no previous study has assessed the impact of upper padding on shooting performance, significant changes in performance have been found across difference boot models [2], stance leg traction [10] and toe box height
[8]. However, not controlling the run up and no measure of the ball direction (accuracy), may have impacted the results. Using the applied protocol developed in this study to reassess these design features could strengthen the football boot design knowledge for shooting performance.

Despite the direction of offset being crucial in football, previous literature investigating shooting accuracy has only assessed radial offset $[11,12]$. No significant difference was found for radial or horizontal offset for both accuracy and velocity focused shots between the upper padding conditions. Vertical offset, however, demonstrated significant differences for both accuracy and velocity focused shots with those performed in the non-padded boot being significantly closer to target than those performed in the padded boot for both types of shots. This also resolved in more misses above goal for the padded boot. For the nonpadded boot $57-8 \%$ of shots went inside the goal (zone 3 ) and $30 \%$ directly above goal (zone 1), while for the padded boot $28-44 \%$ went inside goal and $43-56 \%$ directly above. These differences would not have been detected by solely assessing radial offsets. These results indicate players were challenged to keep the ball down and on target with the added padding which fails to lend support to accuracy or ball velocity performance benefits from added padding to football boot design. While an adaptation period may alter these results, the initial 'try on' results of this study do not favour added padding to football boots for shooting performance. Similar to velocity measures, studies have detected significant differences in radial offset with other design features (boot model [11] and lacing style [12]). Yet, comparable to the velocity focused studies the abovementioned external factors and lack of ball velocity control may have impacted these results.

No previously published research has measured both shot velocity and accuracy within the same study. This should be
Table 2 Success comparison results for the $0 \mathrm{~mm}$ and $8 \mathrm{~mm}$ padded boots in session 1 and relative and absolute test-retest reliability scores for repeated $0 \mathrm{~mm}$ padded boot test sessions

\begin{tabular}{llllllllll}
\hline Skill & Boot & Success & $\%$ & $P$ value & $\begin{array}{l}\text { Session 1 } \\
\text { Mean } \pm \text { SD }\end{array}$ & $\begin{array}{l}\text { Session 2 } \\
\text { Mean } \pm \text { SD }\end{array}$ & ICC $_{2,1}$ & SEM & SRD \\
\hline Accuracy kick & $0 \mathrm{~mm}$ & Goal & 58 & $0.001^{* * *}$ & $58 \pm 21$ & $58 \pm 26$ & 0.925 & \pm 4 & \pm 12 \\
& & No goal & 41 & & & & & & \\
& $8 \mathrm{~mm}$ & Goal & 28 & & & & & & \\
& & No goal & 72 & & & & & & \\
Velocity kick & $0 \mathrm{~mm}$ & Goal & 57 & $0.001^{* * *}$ & $57 \pm 22$ & $55 \pm 17$ & 0.527 & \pm 7 & \pm 20 \\
& & No goal & 43 & & & & & & \\
& \multirow{2}{*}{$8 \mathrm{~mm}$} & Goal & 44 & & & & & & \\
& & No goal & 56 & & & & & & \\
\end{tabular}

Pearson's $\mathrm{Chi}^{2}$ test

$I C C_{2,1}$ intraclass correlation coefficient: two-way random effect model (absolute agreement definition), $S D$ standard deviation, SEM Standard error of measurement $=\mathrm{SD} \times \sqrt{1-\mathrm{ICC}}, S R D$ smallest real difference at $95 \%$ confidence intervals $=\mathrm{SEM} \times 1.96 \times \sqrt{2}$

$P$ value significance level set at 0.05 

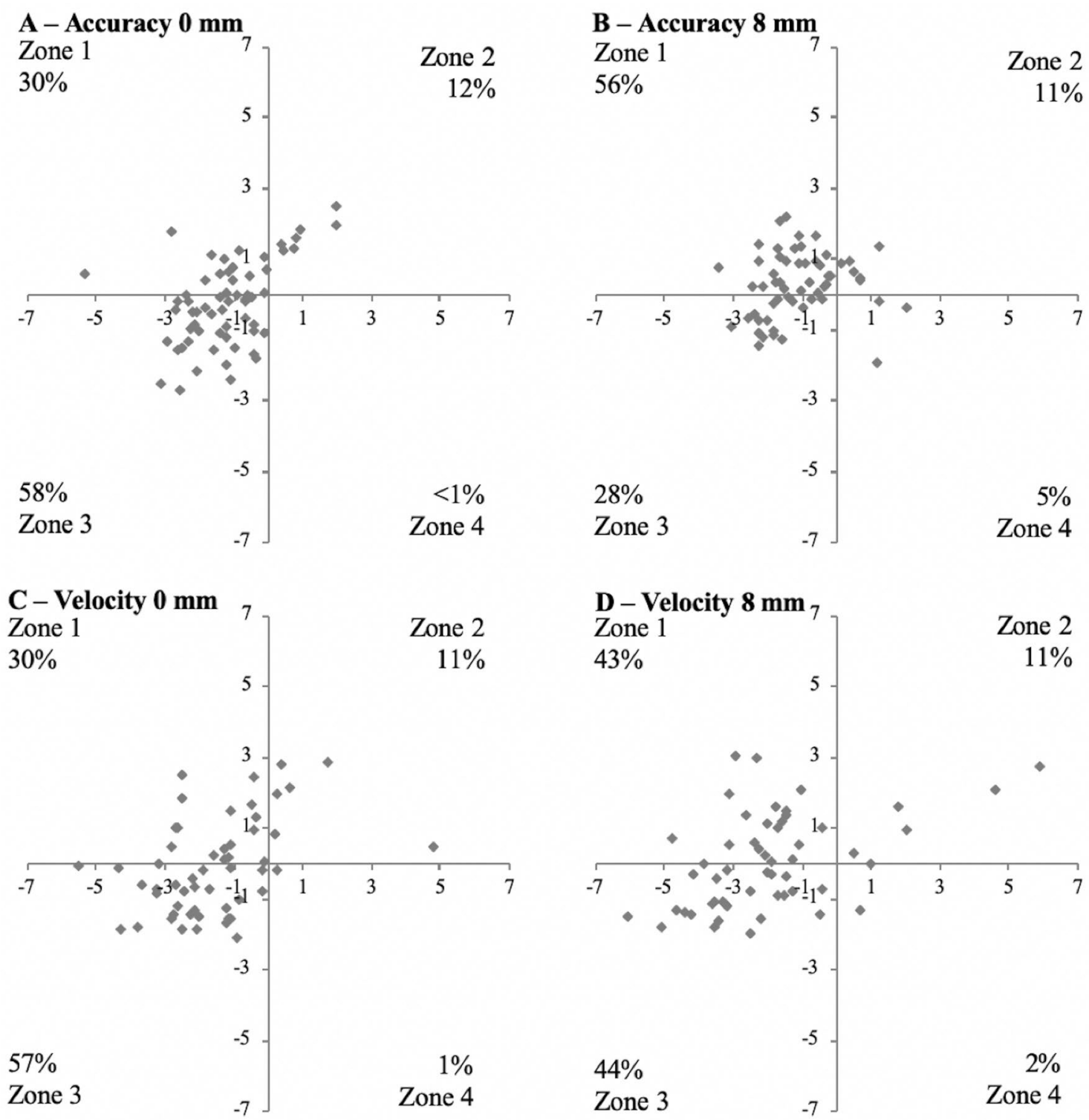

Fig. 4 a-d Shot outcome distribution for accuracy and velocity shots split by upper padding condition in session 1 with percentage of shots within the four designated zones around the target point. $(0,0)$ represents the target point

considered a limitation as players may alter one to gain the desired performance for the other, thereby confounding the outcome. The speed-accuracy trade-off [13] was observed in this study; a velocity focus led to higher shot velocities but with a reduced shot accuracy and repeatability, while an accuracy focus led to more accurate shots but ball velocity was lower and less repeatable.

The test-retest reliability for ball velocity and accuracy is important to understand, as human testing is likely to be impacted by external factors such as player skill level and equipment sensitivity. Assessments in football have previously been conducted for shooting performance focused studies. These demonstrated lower reliability than observed in this study despite similar shooting distances being used (16 $\mathrm{m}$ in this study, $15 \mathrm{~m}$ in Russell et al. [16] and $16.5 \mathrm{~m}$ in Ali et al. [15]). Differences may lie within the player ability, run up, shot type and ball impact point, which have all been shown to impact shooting performance [23-25, 30-35]. Ali et al. [15] and Russell et al. [16] assessed protocols designed to investigate player skill level on in-play shooting performance and, therefore, included less control for consistency in the shooting task; this study focused on a dead ball situation with greater control over the shooting task. Therefore, for this study it was necessary to develop a novel test specific protocol. The test-retest reliability values obtained indicate that the proposed protocol is appropriate when assessing 
Table 3 Zonal offset results for the $0 \mathrm{~mm}$ and $8 \mathrm{~mm}$ padded boots in session 1 and relative and absolute test-retest reliability scores for repeated $0 \mathrm{~mm}$ padded boot test sessions

\begin{tabular}{|c|c|c|c|c|c|c|c|c|c|}
\hline Skill & Boot & Zone & $\%$ & $P$ value & $\begin{array}{l}\text { Session } 1 \\
\text { Mean } \pm \text { SD }\end{array}$ & $\begin{array}{l}\text { Session } 2 \\
\text { Mean } \pm \text { SD }\end{array}$ & $\mathrm{ICC}_{2,1}$ & SEM & SRD \\
\hline \multirow[t]{8}{*}{ Accuracy kick } & \multirow[t]{4}{*}{$0 \mathrm{~mm}$} & 1 & 30 & \multirow[t]{8}{*}{$0.006^{* *}$} & $30 \pm 17$ & $25 \pm 17$ & 0.603 & \pm 6 & \pm 17 \\
\hline & & 2 & 12 & & $12 \pm 13$ & $16 \pm 15$ & 0.460 & \pm 5 & \pm 14 \\
\hline & & 3 & 58 & & $58 \pm 21$ & $58 \pm 26$ & 0.925 & \pm 4 & \pm 12 \\
\hline & & 4 & $<1$ & & $<1 \pm 0$ & $1 \pm 4$ & 0.000 & \pm 2 & \pm 7 \\
\hline & \multirow[t]{4}{*}{$8 \mathrm{~mm}$} & 1 & 56 & & & & & & \\
\hline & & 2 & 11 & & & & & & \\
\hline & & 3 & 28 & & & & & & \\
\hline & & 4 & 5 & & & & & & \\
\hline \multirow[t]{8}{*}{ Velocity kick } & \multirow[t]{4}{*}{$0 \mathrm{~mm}$} & 1 & 30 & \multirow[t]{8}{*}{0.847} & $30 \pm 17$ & $29 \pm 8$ & 0.377 & \pm 7 & \pm 19 \\
\hline & & 2 & 11 & & $11 \pm 14$ & $16 \pm 13$ & 0.471 & \pm 5 & \pm 14 \\
\hline & & 3 & 57 & & $57 \pm 22$ & $55 \pm 17$ & 0.527 & \pm 7 & \pm 20 \\
\hline & & 4 & 1 & & $1 \pm 4$ & $<1 \pm 0$ & 0.000 & \pm 2 & \pm 7 \\
\hline & \multirow[t]{4}{*}{$8 \mathrm{~mm}$} & 1 & 43 & & & & & & \\
\hline & & 2 & 11 & & & & & & \\
\hline & & 3 & 44 & & & & & & \\
\hline & & 4 & 2 & & & & & & \\
\hline
\end{tabular}

Pearson's $\mathrm{Chi}^{2}$ test

$I C C_{2,1}$ intraclass correlation coefficient: two-way random effect model (absolute agreement definition), $S D$ standard deviation, $S E M$ standard error of measurement $=\mathrm{SD} \times \sqrt{1-\mathrm{ICC}}, S R D$ smallest real difference at $95 \%$ confidence intervals $=\mathrm{SEM} \times 1.96 \times \sqrt{2}$

$P$ value significance level set at 0.05 the impact of an external factor, such as boot design or ball design, on shooting performance in football.

In this study, inconsistencies resulting in reduced test-retest reliability were believed to be predominantly caused by the inability of the player to reproduce a consistent shooting output. Ball velocity was more reliable for velocity focused shots $\left(S R D \pm 1.2 \mathrm{~m} \mathrm{~s}^{-1}\right)$ in comparison to accuracy focused shots $\left(\mathrm{SRD} \pm 3.2 \mathrm{~m} \mathrm{~s}^{-1}\right)$ most likely due to the lesser attention given to ball velocity for the latter shot type. Similar trends were seen for shot accuracy. This indicates that when a player is asked to focus on a specific outcome variable, e.g., maximise shot velocity, the natural human variation in this outcome variable is reduced.

\subsection{Limitations}

No boot 'break in' experience or longer-term adaptation period for players was included within the test protocol. It should be acknowledged that both short- and long-term changes in performance may occur when a player is exposed to new football boots although neither were the focus of this study.

Second, when developing a protocol to assess the impact of a small adjustment like football boot design then it is essential to control for other confounding factors. Within this study the aim was to allow natural player movement in a repeatable pattern while also including more control than past literature investigating the effect of boot design on shooting performance specifically in the run-up phase. Players reported that once their preferred run up had been set, the shooting appeared to be a natural movement for them. This is expected, since players' practice free kicks in training which includes trying to achieve an optimal run up pattern. By controlling run up and shot type it is believed that ecological validity can be achieved despite actively trying to minimise human error.

Third, the technical level of the players recruited will impact the outcome of human testing. Higher technical level leads to reduced intra-participant variability in technique $[36,37]$. This study assessed skilled university players and future research should aim to include participants at an equivalent or higher level to maintain reliability scores within the values obtained in this study.

Finally, only one boot design, padding material, padding thickness and padding area was tested. Further study is required to assess the degree to which the results obtained herein are applicable across a greater range in these variables. 


\section{Conclusion}

In summary, a novel protocol has been developed to assess the impact of equipment, e.g., football boots, on performance of shooting measured though ball velocity and ball accuracy measures. Comparing two boot models with different upper padding thickness (no additional padding and $8 \mathrm{~mm}$ of memory foam padding) a negative impact of upper padding on accuracy has been demonstrated. Specifically, the padding increased the vertical offset of ball flight leading to the player missing the goal more frequently. In contrast, upper padding had no effect on ball velocity. Little is known about how football boot design impacts shooting performance; future research could benefit from the protocol developed herein to reliably investigate this topic.

Acknowledgements We gratefully acknowledge the assistance of Umbro for providing the football boots used in this study and $\mathrm{Mr}$ Jake Warner and Mr Kumbirai Mutamba for assistance during data collection.

Open Access This article is licensed under a Creative Commons Attribution 4.0 International License, which permits use, sharing, adaptation, distribution and reproduction in any medium or format, as long as you give appropriate credit to the original author(s) and the source, provide a link to the Creative Commons licence, and indicate if changes were made. The images or other third party material in this article are included in the article's Creative Commons licence, unless indicated otherwise in a credit line to the material. If material is not included in the article's Creative Commons licence and your intended use is not permitted by statutory regulation or exceeds the permitted use, you will need to obtain permission directly from the copyright holder. To view a copy of this licence, visit http://creativecommons.org/licenses/by/4.0/.

\section{References}

1. Xerfi 2XDIS04 (2015) Sporting goods companies-world. In: Market analysis-2013-2015 trends-corporate strategies. Groupe Xerfi; 2015

2. Hennig EM, Zulbeck O (1999) The influence of soccer boot construction on ball velocity and shock to the body. In: Fourth Symp. Footwear Biomech., Canmore, Canada: Footwear Biomechanics; 1999, pp 52-53

3. Ismail AR, Ali MFM, Deros BM, Rashid MSA (2010) Comparative study of kicking performance based on different kind of shoes. In: Rahman Al MM (Eds) Natl. Conf. Mech. Eng. Res. Postgrad. Stud., Kuanton, Pohang, Malaysia: 2010, pp 275-279

4. Sterzing T, Kroiher J, Hennig E (2006) Barefoot vs. shod kicking in soccer-what's faster? J Biomech 39:551. https://doi.org/10. 1016/S0021-9290(06)85268-1

5. Taha Z, Aris MA, Hassan MHA (2013) The influence of football boot construction on ball velocity and deformation. IOP Conf Ser Mater Sci Eng 50:012028. https://doi.org/10.1088/1757-899X/ 50/1/012028

6. Amos M, Morag E (2002) Effect of shoe mass on soccer kicking velocity. In: Proc. 4th World Congr. Biomech., Calgary, Alberta, Canada: 2002
7. Moschini A, Smith N (2012) Effect of shoe mass of soccer kicking velocity. In: ISBS-Conf. Proc. Arch., Melbourne, Australia: 2012, p. $150-153$

8. Sterzing T, Hennig EM (2008) The influence of soccer shoes on kicking velocity in full-instep kicks. Exerc Sport Sci Rev 36:9197. https://doi.org/10.1097/JES.0b013e318168ece7

9. Sterzing T, Hennig EM. The influence of friction properties of shoe upper materials on kicking velocity in soccer. In: 21 Congr Int Soc Biomech 2007;40 (Supplement 2):195. https://doi.org/10. 1016/S0021-9290(07)70191-4

10. Sterzing T, Hennig EM (2007) The influence of stance leg traction properties on kicking performance and perception parameters in soccer. In: 8th Footwear Biomech. Symp. 8th Footwear Biomech. Symp., Taipei, Taiwan: 2007, p. 27-8

11. Hennig EM, Althoff K, Hoemme A-K (2009) Soccer footwear and ball kicking accuracy. Footwear Sci 1(S1):85-87

12. Kuo XL, Shiang T-Y (2007) The instep kicking accuracy analysing different soccer shoes. J Biomech 40(s2):629

13. Fitts PM (1954) The information capacity of the human motor system in controlling the amplitude of movement. J Exp Psychol 47:381-391

14. Okholm Kryger K, Mitchell SR, Forrester S. The speed-accuracy trade-off for football kicks. In: UK Footwear Sci Meet Stoke Trent 2016

15. Ali A, Williams C, Hulse M, Strudwick A, Reddin J, Howarth L et al (2007) Reliability and validity of two tests of soccer skill. J Sports Sci 25:1461-1470. https://doi.org/10.1080/0264041060 1150470

16. Russell M, Benton D, Kingsley M (2010) Reliability and construct validity of soccer skills tests that measure passing, shooting, and dribbling. J Sports Sci 28:1399-1408. https://doi.org/ $10.1080 / 02640414.2010 .511247$

17. Scurr J, Hall B (2009) The effects of approach angle on penalty kicking accuracy and kick kinematics with recreational soccer players. J Sports Sci Med 8:230-234

18. Elliot K (2014) Umbro UX-1/ Unboxing, Hands-On \& First Impressions. Footy Boots 2014. https://www.footy-boots.com/ umbro-ux-1-unboxing/. Accessed 27 Jan 2020

19. Okholm Kryger K, Mitchell S, Zhou D, Forrester S (2018) The effect of football boot upper padding on dribbling and passing performance using a test-retest validated protocol. Sports Eng 21:401-408. https://doi.org/10.1007/s 12283-018-0284-z

20. Fédération Internationale de Football Association (2015) FIFA quality concept for football turf. Retrieved from http://www.fifa. $\mathrm{com} / \mathrm{mm} /$ document/afdeveloping/pitchequip/fqc_football_turf_ folder_342.pdf. Accessed 5 Aug 2016

21. Neilson PJ (2003) The Dynamic Testing of Soccer Balls. PhD Thesis, 2003

22. Neilson PJ, Jones R (2005) Dynamic soccer ball performance measurement. In: Reilly T, Cabri Arau' JJ (eds) Sci. Footb., vol 5. Routledge, London, pp 21-27

23. Kellis E, Katis A, Gissis I (2004) Knee biomechanics of the support leg in soccer kicks from three angles of approach. Med Sci Sports Exerc 36:1017-1028

24. Kellis E, Katis A (2007) Biomechanical characteristics and determinants of instep soccer kick. J Sports Sci Med 6:154-165

25. Sakamoto K, Geisler G, Nakayama M, Asai T (2011) Kinematic analysis of the ball impact in female soccer players. Proc Eng 13:182-187. https://doi.org/10.1016/j.proeng.2011.05.070

26. Okholm Kryger K, Mitchell S, Forrester S (2019) Assessment of the accuracy of different systems for measuring football velocity and spin rate in the field. Proc Inst Mech Eng Part P J Sports Eng Technol 233:324-330. https://doi.org/10.1177/1754337119 830249

27. Weir JP (2005) Quantifying test-retest reliability using the intraclass correlation coefficient and the SEM. J Strength Cond Res 
Natl Strength Cond Assoc 19:231-240. https://doi.org/10.1519/ 15184.1

28. Cicchetti DV (1994) Guidelines, criteria, and rules of thumb for evaluating normed and standardized assessment instruments in psychology. Psychol Assess 6:284-290

29. Vaz S, Falkmer T, Passmore AE, Parsons R, Andreou P (2013) The case for using the repeatability coefficient when calculating test-retest reliability. PLoS One. https://doi.org/10.1371/journ al.pone. 0073990

30. Asai T, Carre M, Akatsuka T, Haake SJ (2002) The curve kick of a football I: impact with the foot. Sports Eng 5:183-192

31. Hong S, Go Y, Sakamoto K, Nakayama M, Asai T (2013) Characteristics of ball impact on curve shot in soccer. Proc Eng 60:249254. https://doi.org/10.1016/j.proeng.2013.07.008

32. Hong S, Kazama Y, Nakayama M, Asai T (2012) Ball impact dynamics of knuckling shot in soccer. Proc Eng 34:200-205. https://doi.org/10.1016/j.proeng.2012.04.035

33. Levanon J, Dapena J (1998) Comparison of the kinematics of the full-instep and pass kicks in soccer. Med Sci Sports Exerc 30:917-927

\section{Authors and Affiliations}

\section{Katrine Okholm Kryger ${ }^{1}$ (D) . Séan Mitchell ${ }^{2,3}$ (D) $\cdot$ Diwei Zhou $^{4}\left(\mathbb{D} \cdot\right.$ Steph Forrester $^{2,3}(\mathbb{D}$}

Séan Mitchell

S.R.Mitchell@lboro.ac.uk

Diwei Zhou

D.Zhou2@lboro.ac.uk

Steph Forrester

S.Forrester@lboro.ac.uk

1 Sport and Exercise Medicine, Queen Mary University of London, London E1 4NS, UK
34. Nunome H, Asai T, Ikegami Y, Sakurai S (2002) Three-dimensional kinetic analysis of side-foot and instep soccer kicks. Med Sci Sports Exerc 34:2028-2036. https://doi.org/10.1249/01.MSS. 0000039076.43492.EF

35. Sakamoto K, Geisler G, Nakayama M, Asai T (2010) Kinematics of the foot joint in female soccer players during the ball impact phase of kicking. Proc Eng 2:2549-2554. https://doi.org/10. 1016/j.proeng.2010.04.030

36. Anderson DI, Sidaway B (1994) Coordination changes associated with practice of a soccer kick. Res Q Exerc Sport 65:93-99. https://doi.org/10.1080/02701367.1994.10607603

37. Manolopoulos E, Papadopoulos C, Kellis E (2006) Effects of combined strength and kick coordination training on soccer kick biomechanics in amateur players. Scand J Med Sci Sports 16:102110. https://doi.org/10.1111/j.1600-0838.2005.00447.x

Publisher's Note Springer Nature remains neutral with regard to jurisdictional claims in published maps and institutional affiliations.
2 School of Mechanical, Electrical and Manufacturing Engineering, Loughborough University, 1 Oakwood Dr, Loughborough LE11 3QF, Leicestershire, UK

3 Sports Technology Institute, Loughborough University, 1 Oakwood Dr, Loughborough LE11 3QF, Leicestershire, UK

4 Department of Mathematical Sciences, Loughborough University, Epinal Way, Loughborough LE11 3TU, Leicestershire, UK 